\title{
Single Top and Dark Matter: \\ Two is not always better than one
}

\author{
Alberto Zucchetta* \\ Zurich University \\ E-mail: a.zucchetta@cern.ch
}

To date, only two modes of production at hadron colliders of dark matter through new scalar or pseudoscalar mediators have been considered in the existing literature: pairs of dark matter particles produced through top quark loops with an associated hadronic jet in the event (monojet), and production of dark matter with pairs of heavy flavored top or bottom quarks. We present a third, previously overlooked channel, which consists of dark matter production in association with a single top quark. In spite of a generally lower production cross section at LHC when compared to the associated top-pair channel, non-flavor violating single top quark processes are kinematically favored and can greatly increase the sensitivity to these models. We will show that including dark matter production in association with a single top quark through scalar or pseudoscalar mediators significantly improves the current searches. In particular, the exclusion limit with the available data set by the LHC searches for dark matter and heavy flavors can be substantially improved from $30 \%$ to up to a factor 2 depending on the mass assumed for the mediator particle. We expect that, with a dedicated event selection, the single top and dark matter production mode would demonstrate its full potential, and become the leading channel in Run II and future LHC searches.

The European Physical Society Conference on High Energy Physics

5-12 July, 2017

Venice

${ }^{*}$ Speaker. 


\section{Introduction}

The existence of dark matter (DM) has been established through various astrophysical observations $[1,2,3]$. However, to date there is no compelling evidence of a non-gravitational interaction between DM and standard model (SM) particles. In case DM consists of weakly interacting massive particles (WIMPs), it may be possible to produce pairs of DM particles in the proton-proton collisions at the CERN LHC. The DM particles would not leave an observable signal in the detector; however, if these particles recoil against SM particles, they may produce a large transverse momentum imbalance $\left(\mathscr{E}_{\mathrm{T}}\right)$ in a collision event.

The results of the DM searches are interpreted in terms of simplified Dark Matter models that assume a massive spin-0 (scalar or pseudoscalar) or spin-1 (vector or axial vector) particle that mediates the interaction between DM and SM particles. The parameters of these simplified models include the DM particle mass $\left(m_{\chi}\right)$, the mediator mass $\left(m_{\phi}\right)$, the strength of the coupling between the mediator and SM quarks $\left(g_{\phi}\right)$, and the strength of the coupling between the mediator and DM particles $\left(g_{\chi}\right)$. Reasonable benchmark values for $m_{\phi}$ and $m_{\chi}$ are set in the LHC Dark Matter Working Group recommendations [4]. In the minimal flavor violation hypothesis [5], the spin-0 mediators are assumed to couple to the SM quarks through SM-like Yukawa interactions, proportional to the quark mass, motivating experimental searches for dark matter production in association to heavy-flavored quarks (top and bottom) [6, 7]. In LHC Run II, the final states involving b quarks $[8,9]$ and top quark pair final states $[10,11]$ have been investigated by ATLAS and CMS.

However, a third, previously overlooked channel yields a sizable contribution that should be accounted for in heavy flavor searches. In spite of the generally lower cross sections, single top quark processes have a different production mode and kinematics, resulting in overall rates comparable to top quark pair associated production, especially for a large mediator mass. As a consequence, the sensitivity of the LHC searches can be significantly improved with respect to the searches published by ATLAS and CMS [12]. In this study, the CMS analysis looking for DM production in association with top quark pairs is considered as a reference, and the improvement obtained by adding the single top quark signal on top of the top quark pair production is evaluated.

\section{Generation}

The signal processes are simulated at tree level with the MG5_AMC@NLO v2.4.3 generator [13], and the Pythia v6.428 software [14] is used for showering and hadronization. The $t \bar{t}+\mathrm{DM}$ and $t+\mathrm{DM} t W$-channel processes are produced in the 5-flavor scheme, whereas the 4flavor scheme is used for the $t+\mathrm{DM} t-$ and $s-$ channel processes. For each mass point and process, $200 \mathrm{k}$ events have been generated, except for $t+\mathrm{DM} t$-channel for which this number is increased to 500k. Cross sections at generator level are calculated in 5-flavor scheme for all processes [4], and are presented graphically in Figure 1 for the hypothesis $m_{\chi}=1 \mathrm{GeV}$.

The response of the CMS detector is simulated with DELPHES v3.3.3 [15], using the same data-taking conditions, reconstruction techniques, and efficiencies as in CMS during 2015. The multiplicity of the reconstructed number of leptons, jets, and $b$ tagged jets are presented in Figure 2 for a dark matter particle of mass $1 \mathrm{GeV}$ and a scalar mediator mass of $100 \mathrm{GeV}$. These distributions infer that the phase space used for top quark pair searches may not be optimal for the $t+\mathrm{DM}$ 

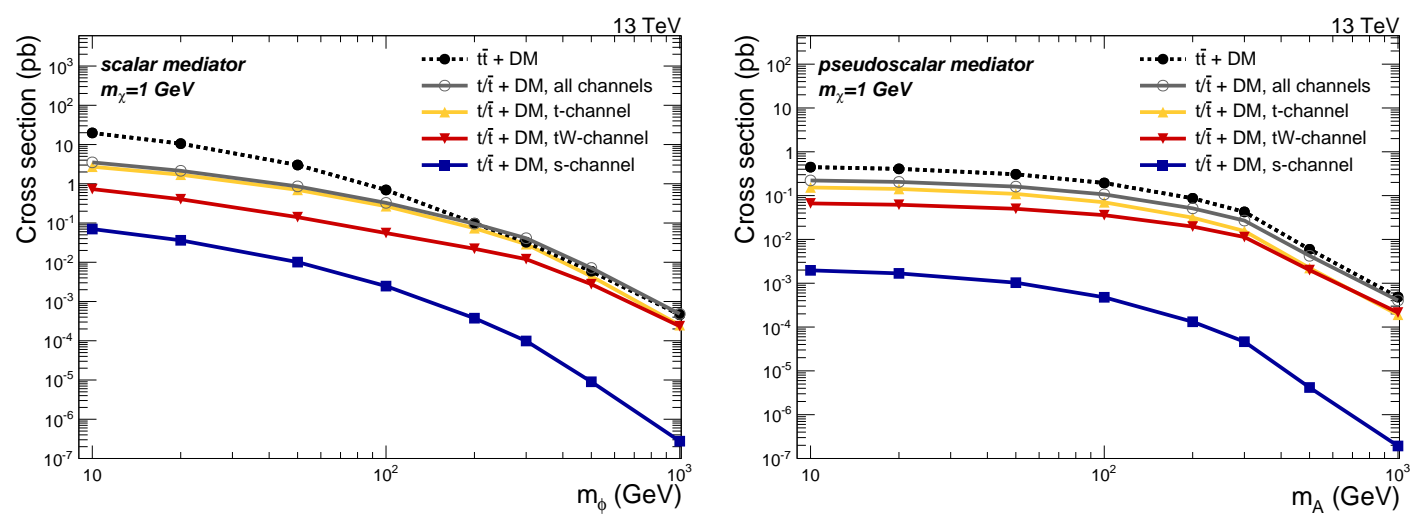

Figure 1: Cross sections of the $t+\mathrm{DM}$ and $t \bar{t}+\mathrm{DM}$ processes for the scalar (left) or pseudoscalar (right) hypothesis assuming different mediator masses and $m_{\chi}=1 \mathrm{GeV}$. The $t+\mathrm{DM}$ processes are split by production mode ( $t-, s-$, and $t W$ channels), and the sum of the three channels is also reported.

signal. The latter has a generally lower jet and $\mathrm{b}$ jet multiplicity, and harder $p_{\mathrm{T}}$ spectra for the jets and leptons. The difference is caused by the presence of just one genuine $\mathrm{b}$ quark in the $t+\mathrm{DM}$ final state, instead of two $\mathrm{b}$ quarks as for the $t \bar{t}+\mathrm{DM}$ signal. The harder $p_{\mathrm{T}}$ spectrum is due to the difference between the initial states: while $t \bar{t}+\mathrm{DM}$ is primarily glue-glue initiated, $t+\mathrm{DM}$ production modes involve quarks, whose parton distribution functions drop less rapidly at large parton momentum fraction.
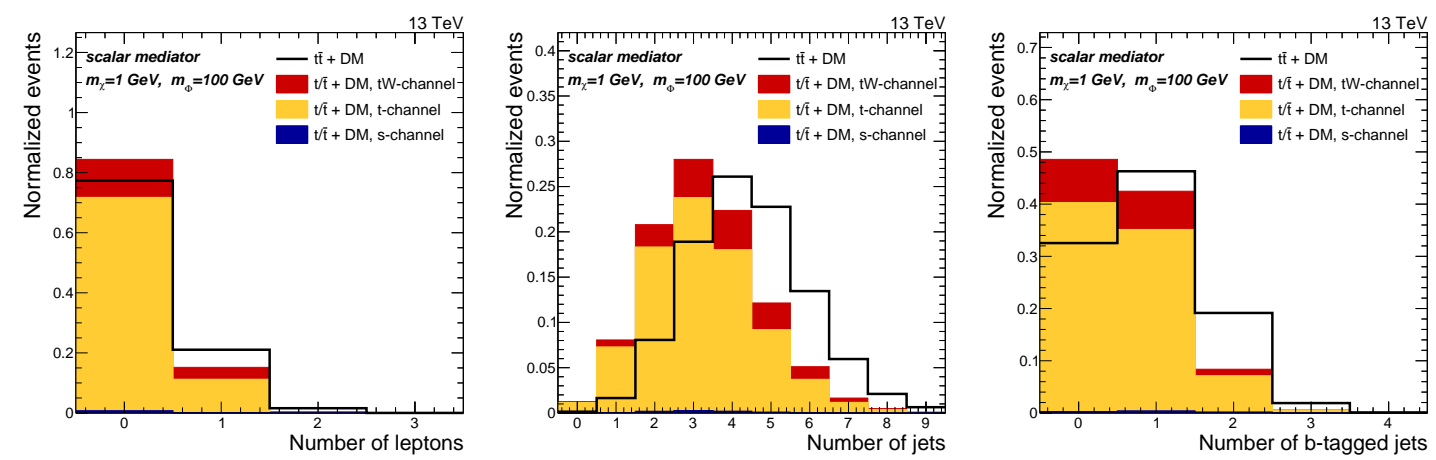

Figure 2: Normalized distributions of the number of reconstructed leptons (left), jets (center), and b tagged jets (right) before any selection for a scalar mediator with mass of $100 \mathrm{GeV}$ and a $1 \mathrm{GeV}$ dark matter particle. The solid black line shows the $t \bar{t}+\mathrm{DM}$ process, while the solid histograms are stacked and show the contribution of the $t+\mathrm{DM}$ processes ( $t W$-channel, $t$-channel, and $s$-channel), weighted by the corresponding cross-section.

\section{Event Selection}

Events are split in two exclusive categories according to the selections in the $t \bar{t}+$ DM CMS analysis [10], depending on the number of leptons in the final state. The zero-lepton, or all-hadronic final state corresponds to the hadronic decay of both top quarks, while the one-lepton final state comes from semi-leptonic decay of one $\mathrm{W}$ boson originated from a top quark decay. The selections 
applied, reported in Table 1, are not the same in the two cases due to the different background composition and trigger requirements. The number of signal events passing the selections are presented graphically in Figure 3 for different mediator mass hypotheses and $m_{\chi}=1 \mathrm{GeV}$.

\begin{tabular}{ccc}
\hline \multirow{2}{*}{ selection } & \multicolumn{2}{c}{ channel } \\
& hadronic & semileptonic \\
\hline $\mathscr{E}_{\mathrm{T}}$ & $>200 \mathrm{GeV}$ & $>160 \mathrm{GeV}$ \\
number of jets & $\geq 4$ & $\geq 3$ \\
number of b-jets & $\geq 2$ & $\geq 1$ \\
number leptons & veto & 1 \\
$p_{T}^{\ell}$ & $>10 \mathrm{GeV}$ & $>30 \mathrm{GeV}$ \\
$\left|\eta^{\ell}\right|$ & $<2.5$ (electrons) & $<2.1$ (electrons) \\
$\Delta \phi\left(j_{i}, \mathbb{E}_{\mathrm{T}}\right)$ & $>2.4$ (muons) & $<2.1$ (muons) \\
$m_{T}^{W}$ & $-i=1, . ., 6$ & $>1.2 ; i=1,2$ \\
$m_{T 2}^{W}$ & - & $>160 \mathrm{GeV}$ \\
\end{tabular}

Table 1: Event selections for the hadronic and semileptonic channels, as applied in the CMS analysis [10].
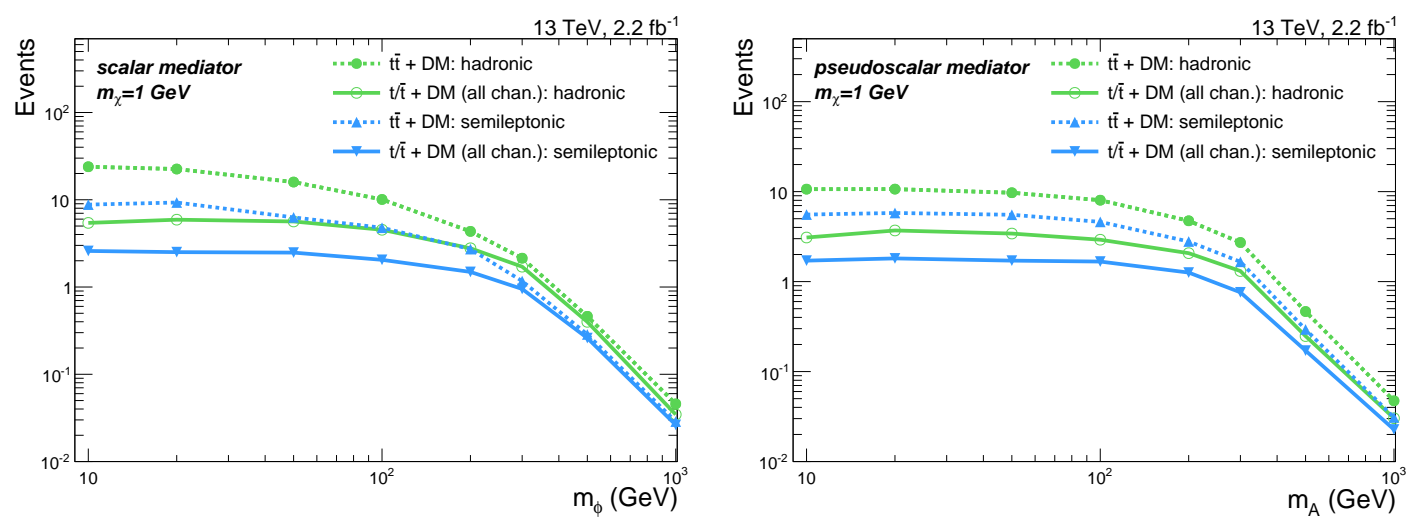

Figure 3: Expected signal events for scalar (left) and pseudoscalar (right) mediators for various $m_{\varphi}$ mass choices and $m_{\chi}=1 \mathrm{GeV}$. The numerical values are referred to an integrated luminosity of $2.2 \mathrm{fb}^{-1}$, and are separated by process $(t \bar{t}+\mathrm{DM}$ or the sum of all $t+\mathrm{DM}$ production channels) and final state (hadronic or semileptonic).

\section{Impact on the exclusion limit}

The sensitivity of the $t+$ DM process is assessed by calculating with a counting experiment the improvement on the final exclusion limits obtained by the inclusion of $t+\mathrm{DM}$ events in addition to the $t \bar{t}+\mathrm{DM}$ signal. The limits are set on the signal strength multiplier $\mu$ with respect to the predicted cross section with $g_{\chi}=g_{v}=1$. The expected number of background events and the corresponding uncertainty are obtained from the CMS analysis [10], and the signal is normalized to the simulated $t+\mathrm{DM}$ and $t \bar{t}+\mathrm{DM}$ processes.

The upper limits, obtained with the CLs method [16] in the asymptotic approximation [17] are shown for the $m_{\chi}=1 \mathrm{GeV}$ assumption in Figure 4. The resulting values for $t \bar{t}+\mathrm{DM}$ alone are found 

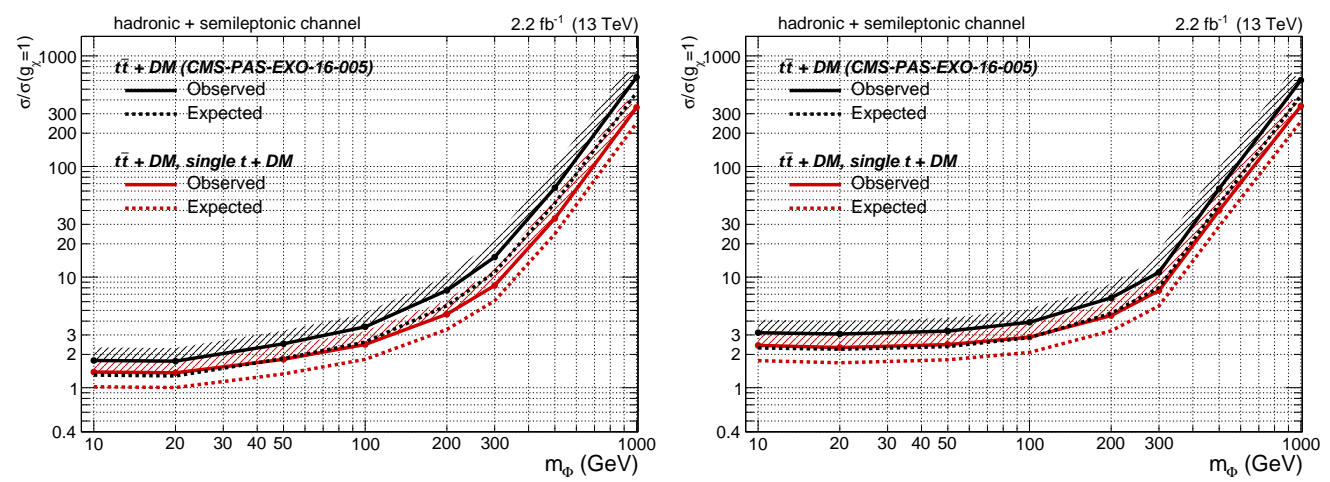

Figure 4: Comparison between the expected and observed exclusion limits on $\mu=\sigma / \sigma_{t h}$ considering the $t \bar{t}+$ DM signal alone as in Ref. [10] (black dotted and solid lines), and with the combined $t \bar{t}+\mathrm{DM}$ and $t+$ DM signals (red dotted and solid lines) for the scalar (left) and pseudoscalar (right) mediator hypothesis. The area above the lines, indicated by the shaded areas, represents the excluded parameter space.
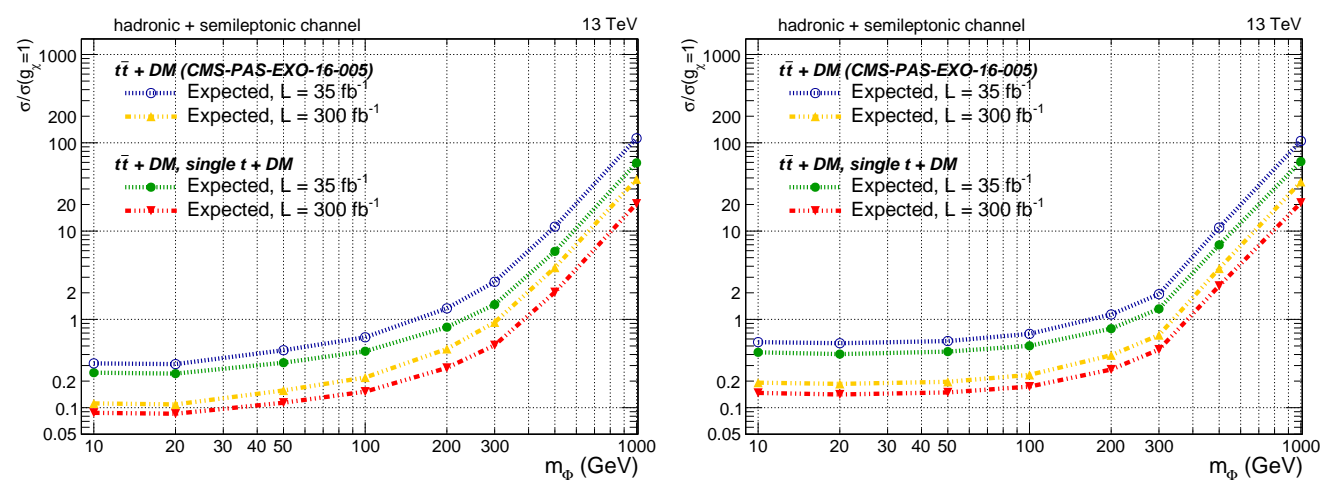

Figure 5: Expected exclusion limits on $\mu=\sigma / \sigma_{t h}$ for the $t \bar{t}+\mathrm{DM}$ signal (blue and yellow lines) and the sum of $t+\mathrm{DM}$ and $t \bar{t}+\mathrm{DM}$ (green and red lines) for the scalar (left) and pseudoscalar (right) mediator hypothesis. The two scenarios considered are for the 2016 dataset $\left(35 \mathrm{fb}^{-1}\right)$ and LHC Run II $\left(300 \mathrm{fb}^{-1}\right)$.

to be comparable to the inclusive CMS analysis [10], and by including the $t+\mathrm{DM}$ processes, the relative improvements in the expected limit ranges from $30 \%$ at low mediator mass up to a factor of two at high mediator mass $\left(m_{\varphi}=1 \mathrm{TeV}\right)$.

The sensitivity of the analysis is also projected for two future scenarios. Considering the dataset collected during 2016 and at the end of LHC Run II, an estimated integrated luminosity of $35 \mathrm{fb}^{-1}$ and $\sim 300 \mathrm{fb}^{-1}$ will be available to the ATLAS and CMS experiments. Without any reliable method to estimate the systematic uncertainties for these datasets, the results are extracted under the assumption that the signal uncertainty is proportional to the integrated luminosity and background uncertainties will scale as the square root of luminosity. The exclusion limits are reported in Figure 5. Even with large luminosities, it is verified that the addition of the $t+\mathrm{DM}$ signal still increases significantly the sensitivity on the exclusion limit. 


\section{Summary}

For the first time, the production of dark matter in association with a single top quark has been considered. Thanks to its larger selection efficiency and the harder transverse momentum spectra of the visible particles, the contribution of this production mode is sizable and should be considered in the next iterations of searches for Dark Matter and heavy flavors.

The contribution has been verified by simulating the $t+\mathrm{DM}$ signal and simulating the response of the CMS detector in order to evaluate the improvement obtainable by adding the new signal to the pre-existing $t \bar{t}+\mathrm{DM}$ signal. The effect on the sensitivity on the exclusion limit is between $30 \%$ and a factor of two. The same improvement is confirmed also for future analyses with the 2016 dataset and the LHC Run II expected luminosity.

\section{References}

[1] G. Bertone et al., Phys. Rept. 405, 279 (2005)

[2] J. L. Feng Ann. Rev. Astron. Astrophys. 48, 495 (2010)

[3] T. A. Porter et al, Ann. Rev. Astron. Astrophys. 49, 155 (2011)

[4] D. Abercrombie et al., arXiv:1507.00966

[5] G. D’Ambrosio, G. F. Giudice, G. Isidori, and A. Strumia, Nucl. Phys. B 645 (2002)

[6] M. R. Buckley, Phys. Rev. D 84 (2011) 043510, arXiv:1104.1429

[7] M. R. Buckley, D. Feld, and D. Goncalves et Al., Phys. Rev. D 91 (2015) 015017, arXiv:1410.6497

[8] CMS Collaboration, CMS-PAS-B2G-15-007 (2016)

[9] ATLAS Collaboration, ATLAS-CONF-2016-086 (2016)

[10] CMS Collaboration, CMS-PAS-EXO-16-005 (2016)

[11] ATLAS Collaboration, Eur. Phys. J. C 75 (2015), arXiv:1410.4031

[12] D. Pinna, A. Zucchetta, M. R. Buckley, F. Canelli, Phys. Rev. D 96 (2017), arXiv:1701.05195, doi:10.1103/PhysRevD.96.035031

[13] J. Alwall et al., JHEP 2014 (2014)

[14] T. Sjostrand, S. Mrenna, and P. Z. Skands, JHEP 05 (2006) 026, arXiv:hep-ph/0603175

[15] J. de Favereau et al., JHEP 02 (2014) 057, arXiv:1307.6346

[16] A. L. Read, J. Phys. G 28 (2002)

[17] G. Cowan, K. Cranmer, E. Gross, and O. Vitells, Eur. Phys. J. C 71 (2011) 1554, arXiv:1007.1727, [Erratum: doi:10.1140/epjc/s10052-013-2501-z] 